NOTAS

\title{
Efecto del ácido indolbutírico en la capacidad rizogénica de estacas de Eucryphia glutinosa
}

\author{
Indole butyric acid effect on rhizogenic capacity of Eucryphia glutinosa cuttings
}

\author{
Mirtha Latsague Vidala ${ }^{*}$, Patricia Sáez Delgado ${ }^{a}$, Jéssica Yáñez Delgado ${ }^{a}$ \\ *Autor de correspondencia: aUniversidad Católica de Temuco, Escuela de Ciencias Ambientales, \\ Facultad de Recursos Naturales, casilla 15-D, tel.: (45)205409, fax: (45)211034, Temuco, Chile, mlatsagu@uct.cl
}

\begin{abstract}
SUMMARY
Eucryphia glutinosa is an endemic species from Chile that at the present is with conservation problems, being catalogued as a rare species. Its distribution area and its population are decreasing considerably due to deforestation. The objective of the present work was to evaluate its reproductive capacity by means of cuttings rhizogenesis. Cuttings were collected during April 2007 in the National Malleco Reserve, Araucania Region. The harvested cuttings were treated with indole butyric acid (IBA) at different concentrations $\left(0,250,500,1,000\right.$ and $\left.1,500 \mathrm{mg} \mathrm{L}^{-1}\right)$ as rooting stimulator. After seven months in a hot bed a $30 \%$ of total rooting was obtained. The best results in respect of the process of rhizogenesis were shown by $500 \mathrm{mg} \mathrm{L}^{-1}$ IBA, with an average of $3 \mathrm{~cm}$ in root length and 12 roots by cutting, with a $56.5 \%$ of rooting.
\end{abstract}

Keys word: Eucryphia glutinosa, cuttings, adventitious rhizogenesis, IBA

RESUMEN

Eucryphia glutinosa es una especie endémica de Chile que actualmente se encuentra con problemas de conservación, siendo catalogada como especie rara. Su área de distribución y su contingente poblacional han disminuido considerablemente, por lo que aplicar protocolos de propagación resulta necesario para aumentar el número de individuos. Actualmente existe escasa información respecto a la reproducción vegetativa de esta especie. En consideración a esto, el objetivo del trabajo fue evaluar su capacidad reproductiva mediante rizogénesis de estacas. Se colectaron estacas en abril de 2007 en la Reserva Nacional Malleco, Región de La Araucanía. Las estacas fueron tratadas con ácido indolbutírico (AIB) durante 15 minutos en distintas concentraciones $(0,250,500,1.000$ y $1.500 \mathrm{mg} \mathrm{L}^{-1}$ ). Después de mantener las estacas durante siete meses en cama caliente de arraigamiento, se evaluaron los porcentajes de sobrevivencia, de formación de callo y de enraizamiento. Además, se determinó el potencial de crecimiento radical (PCR). Se observó que los mejores resultados se produjeron en la concentración de $500 \mathrm{mg} \mathrm{L}^{-1}$ de AIB, con un $56,5 \%$ de enraizamiento.

Palabras clave: Eucryphia glutinosa, estacas, rizogénesis adventicia, AIB.

\section{INTRODUCCIÓN}

La conservación de especies y de los ecosistemas forestales que éstas conforman es importante dado su valor como reserva genética (Wilken 1998). Eucryphia glutinosa (Poepp. et Endl.) Baillon (guindo santo) es una especie endémica de Chile que se distribuye en la Cordillera de Los Andes, entre las regiones del Maule y La Araucanía (Gajardo 1986), y está presente en los tipos forestales Ciprés de la Cordillera, Roble-Hualo y Roble-RaulíCoigüe (Hechenleitner et al. 2005). No obstante que en forma natural ocupa una gran área geográfica, presenta muy bajas densidades poblacionales y se encuentra catalogada como especie rara (Benoit 1989). Su problema de conservación está asociado a la construcción de represas hidroeléctricas, la deforestación y la corta de bosque nativo para plantaciones y propósitos agrícolas (Hechenleitner et al. 2005). La extinción de esta especie sería una enorme pérdida, tanto desde el punto de vista de la biodiversidad como patrimonial. Esta especie posee un importante potencial económico debido, principalmente, al colorido de su follaje y sus grandes flores blancas de gran valor ornamental y melífera (Hechenleitner et al. 2005). Los contingentes poblacionales de E. glutinosa son escasos, los que se podrían aumentar a través de su reproducción. La reproducción sexual de esta especie no está bien documentada, existiendo controversia respecto a su capacidad germinativa. En cuanto a la reproducción asexual, existe escasa información de propagación por estacas. Se justifica, entonces, comunicar los resultados obtenidos en un ensayo 
de enraizamiento de estacas efectuado en condiciones de invernadero.

Muchos son los factores que pueden ser determinantes en el éxito del arraigamiento de estacas, entre ellos, las auxinas que favorecen la formación de raíces adventicias porque modifican la extensibilidad celular, al producir factores que ablandan la pared. El ácido indolbutírico (AIB) es un fitorregulador auxínico sintético comúnmente utilizado por su estabilidad, ya que es muy resistente a la oxidación por la luz, enzimas u otros agentes (Azcón-Bieto y Talón 2000). Así, el objetivo del trabajo fue evaluar la capacidad reproductiva de E. glutinosa mediante propagación por estacas, utilizando distintas concentraciones hormonales de ácido indolbutírico.

\section{MÉTODO}

Se colectó material de árboles adultos ubicados en la Reserva Nacional Malleco, en la Región de La Araucanía ( $38^{\circ} 8^{\prime} 0^{\prime \prime}$ S, $71^{\circ} 47^{\prime}$ 0"). La recolección del material vegetal se realizó durante el mes de abril de 2007, en el sector las Canogas. Se seleccionaron al azar estacas semileñosas, de la sección media de la copa, de individuos que presentaban tallos con crecimientos sanos y vigorosos. El material vegetal fue trasladado en bolsas plásticas oscuras dentro de contenedores fríos al laboratorio, en donde inmediatamente fueron dimensionadas en estacas de $15 \mathrm{~cm}$ de longitud y $0,3 \mathrm{~cm}$ de diámetro, con presencia de al menos dos yemas visibles y dejando como máximo un par de hojas en el tercio superior de la estaca, con un área foliar promedio de $0,08 \mathrm{dm}^{2}$. Se realizaron cortes basales oblicuos, en un recipiente con agua para evitar la desecación de las estacas durante su manipulación.

El medio de enraizamiento consistió en una mezcla de corteza compostada de pino (Pinus radiata D. Don.) (G-15) de pH 5,5 y perlita en una proporción volumétrica de 2:1. Se utilizaron almacigueras aislapol con cavidades de $10 \mathrm{~cm}$ de profundidad y volumen de $80 \mathrm{~cm}^{3}$, las que fueron llenadas con el sustrato indicado.
Las concentraciones de ácido indolbutírico (AIB) utilizadas fueron $250 \mathrm{mg} \mathrm{L}^{-1}, 500 \mathrm{mg} \mathrm{L}^{-1}, 1.000 \mathrm{mg}$ $\mathrm{L}^{-1}$ y $1.500 \mathrm{mg} \mathrm{L}^{-1}$, más un tratamiento control sin AIB. Las estacas con lesión basal se sumergieron durante 15 minutos en las soluciones correspondientes. La desinfección se realizó mediante la inmersión de las estacas en una solución con fungicida Captan ${ }^{\circledR}$ en concentración de $10 \mathrm{~g} \mathrm{~L}^{-1}$. El cultivo se mantuvo en cama caliente a temperatura constante de $25^{\circ} \mathrm{C}$ durante siete meses. La humedad relativa fue de $66 \%$ y la temperatura ambiental fluctuó entre 15 y $20^{\circ} \mathrm{C}$, la que se controló mediante un sistema de calefacción. Se aplicó riego tres veces al día durante un minuto cada vez, a través de un sistema de aspersores (microjet). Se utilizaron 188 estacas por tratamiento, considerando cada estaca como una réplica, utilizando un diseño completo aleatorio. El efecto de la concentración de AIB en las variables sobrevivencia (\%), formación de callos (\%) y enraizamiento (\%) se determinó con la prueba de Chi-cuadrado $(P=0,05)$ (Zar 1996). Sin embargo, para las variables número total de raíces y longitud de la raíz principal (la de mayor extensión) se realizó un análisis de varianza de un factor $(P=0,05)$. Las diferencias significativas fueron determinadas mediante la prueba de rangos múltiples de Fisher (LSD) con un nivel de confianza de $95 \%$.

\section{RESULTADOS}

En general, el porcentaje de estacas vivas fue alto, sin diferenciarse entre tratamientos $(P=0,89)$ (cuadro 1). La aplicación de AIB en las distintas concentraciones ensayadas tampoco influyó significativamente $(P>0,05)$ en el porcentaje de estacas con formación de callos, en el número de raíces ni en la longitud de éstas (cuadro 1). La proporción de estacas enraizadas tuvo influencia significativa del AIB $(P<0,05)$, observándose que concentraciones hormonales de 500 y $1.000 \mathrm{mg} \mathrm{L}^{-1}$ provocaron mayores porcentajes de enraizamiento (cuadro 1 y figura 1 ). El mayor porcentaje de enraizamiento $(56,5 \%)$ se logró con $500 \mathrm{mg} \mathrm{L}^{-1}$ de AIB.

Cuadro 1. Efecto de la concentración de ácido indolbutírico en la rizogénesis de estacas de E. glutinosa colectadas durante abril de 2007. Medias \pm desviación estándar. Letras distintas indican diferencias significativas entre tratamientos $(P<0,05)$.

Effect of IBA concentration on the rooting capacity of E. glutinosa collected in April, 2007. Standard deviations in parenthesis. Different letters indicate significant differences $(P<0.05)$.

\begin{tabular}{lcccrc}
\hline $\begin{array}{c}\text { Tratamiento. } \\
\text { Dosis de AIB* } \\
\left(\mathrm{mg} \mathrm{L}^{-1}\right)\end{array}$ & Sobrevivencia $(\%)$ & $\begin{array}{c}\text { Formación de callo } \\
(\%)\end{array}$ & Enraizamiento (\%) & \multicolumn{2}{c}{ Potencial de crecimiento radical } \\
\hline 0 & $58,7 \pm 21,7 \mathrm{a}$ & $15,3 \pm 2,6 \mathrm{a}$ & $16,9 \pm 10,6 \mathrm{a}$ & $8,4 \pm 3,5 \mathrm{a}$ & $273 \pm 1,1 \mathrm{a}$ \\
250 & $47,2 \pm 10,8 \mathrm{a}$ & $11,5 \pm 1,2 \mathrm{a}$ & $30,7 \pm 5,6 \mathrm{a}$ & $10,0 \pm 6,3 \mathrm{a}$ & $289 \pm 0,3 \mathrm{a}$ \\
500 & $76,7 \pm 9,1 \mathrm{a}$ & $0,0 \pm 0,0 \mathrm{a}$ & $56,5 \pm 10,4 \mathrm{c}$ & $12,0 \pm 9,0 \mathrm{a}$ & $301 \pm 0,3 \mathrm{a}$ \\
1.000 & $67,3 \pm 12,5 \mathrm{a}$ & $6,0 \pm 1,0 \mathrm{a}$ & $40,0 \pm 1,4 \mathrm{~b}$ & $8,4 \pm 8,7 \mathrm{a}$ & $236 \pm 1,5 \mathrm{a}$ \\
1.500 & $61,3 \pm 3,8 \mathrm{a}$ & $18,9 \pm 1,0 \mathrm{a}$ & $21,6 \pm 1,9 \mathrm{a}$ & $5,2 \pm 2,6 \mathrm{a}$ & $220 \pm 0,7 \mathrm{a}$ \\
\hline
\end{tabular}

*AIB: ácido indolbutírico. 


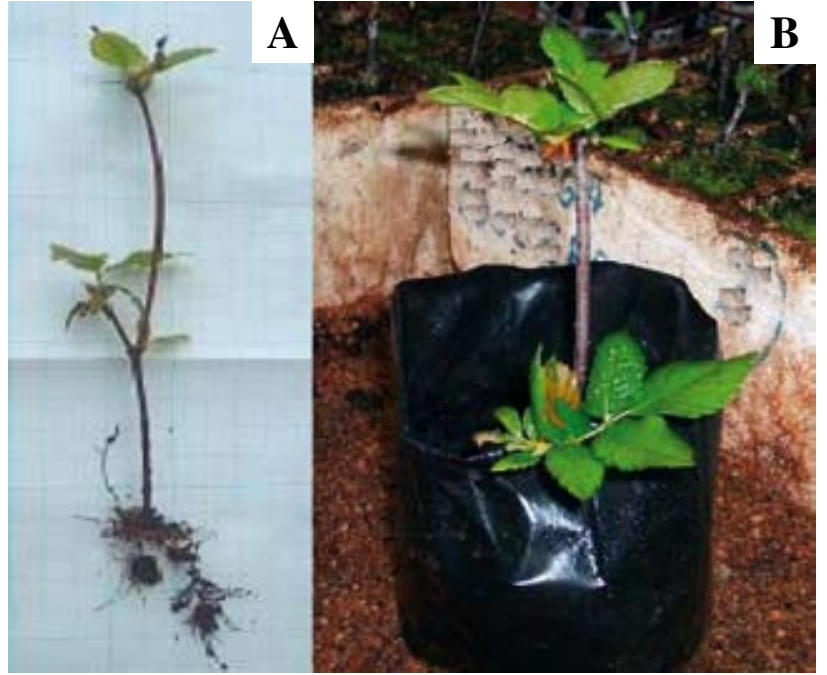

Figura 1. Formación de raíces en estaca de E. glutinosa tratada con $500 \mathrm{mg} \mathrm{L}^{-1}$ de AIB (A) y traspasada posteriormente a bolsa (B).

Eucryphia glutinosa cuttings treated with $500 \mathrm{mg} \mathrm{L}-1$ of IBA (A). Plants of E. glutinosa after seven months of maintaining cuttings under nursery conditions (B).

\section{DISCUSIÓN}

El porcentaje de sobrevivencia total obtenido en E. glutinosa es comparable con resultados obtenidos por Doll et al. (2003), quienes informan un $100 \%$ de sobrevivencia en Buddleja globosa Hope con estacas colectadas en otoño.

Respecto a la aplicación de reguladores de crecimiento, algunos estudios señalan que estacas tratadas con AIB no responden al proceso de rizogénesis (Puig 2003). Sin embargo, el enraizamiento de estacas de E. glutinosa fue influenciado positivamente por la aplicación de AIB en distintas concentraciones. La mejor respuesta en la formación de raíces, obtenida con $500 \mathrm{mg} \mathrm{L}^{-1} \mathrm{de} \mathrm{AIB}$, coincide con lo informado por Ibarra (2000) para esta misma especie, quien obtuvo un $58 \%$ de arraigamiento con igual dosis hormonal. Por su parte, Latsague et al. (2008) al usar la misma concentración hormonal informan 50\% de enraizamiento en Berberidopsis corallina Hook. f. El efecto positivo de concentraciones bajas de AIB coincide con lo informado por Chávez et al. (2000), quienes al trabajar con estacas de Fuchsia magellanica Lam. obtuvieron mejores resultados con concentraciones de 100 y $400 \mathrm{mg} \mathrm{L}^{-1}$ de AIB. Asimismo, Santelices y Cabello (2006) y Santelices (2007) reportan mayores respuestas de enraizamiento en estacas de Nothofagus glauca (Phil.) Krasser en tratamientos con bajas concentraciones de AIB.

A pesar de que la concentración hormonal no influye en el número de raíces formadas, se observa variabilidad con el aumento de la concentración de auxinas. Kozlowski et al. (1991) señalan que la utilización de dosis adecuadas de reguladores de crecimiento es muy importante, puesto que las concentraciones óptimas varían con las especies estudiadas.

La formación de callos en las estacas de E. glutinosa indica que las condiciones en que fueron mantenidas las estacas son adecuadas para el proceso de rizogénesis (Santelices y García 2003). Sin embargo, tal como lo señalan Priestley y Swingle (1929), la formación de callo no necesariamente precede a la de raíces, como queda de manifiesto en la aplicación de hormona $500 \mathrm{mg} \mathrm{L}^{-1}$. Sin embargo, el $100 \%$ de las estacas que formaron callo desarrollan raíces a partir de éste, lo que difiere de la respuesta obtenida en Berberidopsis corallina, la cual, bajo las mismas condiciones de ensayo, sólo forma raíces a partir del tallo (Latsague et al. 2008).

Respecto al potencial de crecimiento radical, los valores de longitud y número de raíces adventicias obtenidos son comparables con los de Santelices y García (2003), quienes informan valores similares al trabajar con estacas de Nothofagus alessandrii Espinosa tratadas con diferentes concentraciones de AIB. En el presente trabajo las variables número y longitud de raíces no dependen de la aplicación de reguladores de crecimiento, sino que serían otros los factores influyentes en ellas, tales como el tipo de sustrato utilizado (Ram et al. 1993), el pH de éste (Santelices y García 2003) u otros. La calificación de la calidad de los sustratos como medio de enraizamiento depende, además de sus características propias, de la especie que se propague en ellos (Gerding et al. 1996). Estos autores señalan que para Podocarpus nubigena Lindl el sustrato de corteza compostada sola o mezclada con otros componentes fue adecuado; en cambio, para Eucryphia cordifolia Cav sólo se consideran adecuados los sustratos de corteza compostada mezclados con otros componentes. Sin embargo, las respuestas obtenidas en E. glutinosa difieren de otros estudios realizados. Es así como Malveira da Silva et al. (2004) informan que al trabajar con estacas de Piper sp. tratadas con distintas concentraciones de AIB se observan diferencias entre los tratamientos en relación al número y longitud de raíces. García et al. (2005) informan un efecto positivo del AIB en el enraizamiento (número y la longitud de las raíces adventicias formadas) de estacas de Gmelina arborea Roxb.

\section{CONCLUSIONES}

Es posible propagar vegetativamente Eucryphia glutinosa a través de estacas semileñosas cosechadas en el mes de abril y tratadas con AIB como factor de enraizamiento. De acuerdo a los resultados obtenidos, en relación al porcentaje de enraizamiento, se recomienda utilizar AIB en concentración de $500 \mathrm{mg} \mathrm{L}^{-1}$ para el estaquillado de E. glutinosa. 


\section{AGRADECIMIENTOS}

Los autores agradecen al Fondo de Protección Ambiental de la Comisión Nacional del Medio Ambiente (FPA CONAMA).

\section{REFERENCIAS}

Azcón-Bieto J, M Talón. 2000. Fundamentos de Fisiología Vegetal. Barcelona, España. Ediciones McGraw-Hill Interamericana. $520 \mathrm{p}$.

Benoit I. 1989. Libro rojo de la flora terrestre de Chile (Primera Parte). Santiago, Chile. CONAF. 157 p.

Chávez W, V Rodríguez, C Boschi, J Molinari, F Vilella. 2000. Respuestas a la aplicación de auxinas y citoquininas en el enraizamiento de estaquillas de Fuchsia magellanica Lam. Buenos Aires, Argentina. $3^{\text {er. }}$ Congreso Argentino de Floricultura. 2 p.

Doll U, H Vogel, P Jeldres, M Muñoz. 2003. Estudios de propagación vegetativa en matico (Buddleja globosa). Ciencia e Investigación Agraria 30: 211-216.

Gajardo R. 1986. Programa de protección y recuperación de la flora nativa de Chile. Ficha técnica de especies amenazadas. Facultad de Ciencias Agrarias y Forestales. Santiago, Chile. Universidad de Chile. 628 p.

García R, J Vargas, V Cetina, A Villegas. 2005. Efecto del ácido indolbutírico (AIB) y tipo de estaca en el enraizamiento de Gmelina arborea. Roxb. Revista Fitotecnología 20: 319-326.

Gerding V, M Hermosilla, R Grez. 1996. Sustratos de corteza compostada para la propagación vegetativa de estacas de tallo de Podocarpus nubigena Lindl. y Eucryphia cordifolia Cav. Bosque 17 (2): 57-64.

Hechenleitner P, M Gardner, P Thomas, C Echeverría, B Escobar. 2005. Plantas amenazadas del Centro-Sur de Chile. Distribución, conservación y propagación. Valdivia, Chile. Universidad Austral de Chile y Real Jardín Botánico de Edimburgo. 188 p.

Ibarra G. 2000. Ecología y propagación de poblaciones de Eucryphia glutinosa (P. et. E) Baillon (Eucryphiaceae). Tesis de Licenciatura. Talca, Chile. Facultad de Ciencias Forestales, Universidad de Talca. 150 p.
Kozlowski T, P Kramer, S Pallardy. 1991. The physiological ecology of woody plants. San Diego, USA. Academic Press. 657 p.

Latsague M, P Sáez, E Hauenstein. 2008. Inducción de enraizamiento en estacas de Berberidopsis corallina con ácido indolbutírico. Bosque 29 (3): 227-230.

Malveira Da Silva J, A Raposo, J Alencar De Sousa, E Melo De Miranda. 2004. Induction of rooting of cuttings of joao-brandinho (Piper sp.) with Indolbuthyric acid. Revista Ciencia Agronómica 35: 248-252.

Priestley J, F Swingle. 1929. Vegetative propagation from the standpoint of the plant anatomy. US Department of Agriculture. 98 p. (Technical Bulletin No 151).

Puig M. 2003. Enraizamiento de estacas de guindo ácido (Prunus cerasus L.), cerezo Santa Lucía (Prunus mahaleb L.) y cerezo dulce (Prunus avium L. cv. Bing). Tesis de Licenciatura. Chillán, Chile. Facultad de Ciencias Agronómicas, Universidad de Concepción. 40 p.

Ram S, S Govind, R Chandra, S Govind. 1993. Efficiency of sugar cane and auxins in relation to rooting and survival of guava layers in acid Alfisol. Progressive Horticulture 23 (1-4): 22-25.

Santelices R, C García. 2003. Efecto del ácido indolbutírico y la ubicación de la estaca en el rebrote de tocón sobre la rizogénesis de Nothofagus alessandrii Espinosa. Bosque 24: 53-61.

Santelices R, A Cabello. 2006. Efecto del ácido indolbutírico, del tipo de la cama de arraigamiento, del substrato, y del árbol madre en la capacidad de arraigamiento de estacas de Nothofagus glauca (Phil.) Krasser. Revista Chilena de Historia Natural 79: 55-64.

Santelices R. 2007. Efecto del ácido indolbutírico (AIB) y la presencia de hojas en el arraigamiento de estacas de Nothofagus glauca (Phil.) Krasser cosechadas en dos épocas diferentes. Revista Ecología Austral 17: 151-158.

Wilken P. 1998. La sustentabilidad forestal en Chile: aporte a una política integral de los bosques nativos y plantaciones exóticas. Santiago, Chile. Serie documentos Experiencias de Cooperación $\mathrm{N}^{\mathrm{o}} 3.62$ p.

Zar JH. 1996. Biostatistical analysis. London, UK. Prentice Hall International. $662 \mathrm{p}$.

Recibido: 19.01 .09

Aceptado: 04.05.09 